\title{
Conducting Polypyrrole/Graphene Nanocomposites as Potential Electromagnetic Interference Shielding Materials in the $K_{u}$-band
}

\author{
Prerna Ramnath Modak, ${ }^{1}$ Deoram Vithoba Nandanwar ${ }^{2}$ and \\ Subhash Baburao Kondawar ${ }^{1 *}$ \\ ${ }^{1}$ Department of Physics, Rashtrasant Tukadoji Maharaj Nagpur University, \\ Amravati Road, Nagpur, Maharashtra 440033, India \\ ${ }^{2}$ Department of Physics, Shri Mathuradas Mohata College of Science, \\ Nagpur, Maharashtra 440009, India \\ ${ }^{*}$ Corresponding author: sbkondawar@yahoo.co.in
}

Published online: 25 November 2016

To cite this article: Modak, P. R. et al. (2016). Conducting polypyrrole/graphene nanocomposites as potential electromagnetic interference shielding materials in the $\mathrm{K}_{\mathrm{u}}$-band. J. Phys. Sci., 27(3), 137-157, http://dx.doi.org/10.21315/jps2016.27.3.9

To link to this article: http://dx.doi.org/10.21315/jps2016.27.3.9

\begin{abstract}
Enormous progress in nanotechnology has made electronic systems smaller but has also created a new type of problem called electromagnetic interference (EMI). Carbon-based conducting polymer nanocomposites have potential applications as EMI shielding materials owing to their high conductivity and dielectric constant of the materials that contribute to the high EMI shielding efficiency (SE). In the present investigation, highly conducting polypyrrole (PPy)/graphene (GNS) nanocomposites were prepared by in-situ polymerisation with different concentration of functionalised GNS (1\%, 3\% and 5\%). UV-VIS and FTIR show a systematic shifting of the characteristic bands of PPy, with the increase in the GNS phase suggesting significant interaction between the phases. The SEM images show thick and uniform coating of PPy over the surface of individual GNS. PPy/GNS nanocomposites showed a semiconducting behaviour similar to that of PPy as well as improved dielectric and EMI shielding properties. The EMI shielding effectiveness (SE) and dielectric constant of nanocomposites were found to increase with increasing GNS content and were found to be absorption-dominated, indicating that PPy/GNS nanocomposites are potential lightweight EMI shielding materials for the protection of electronic systems from electromagnetic radiation in the $K_{u}$-band.
\end{abstract}

Keywords: Nanocomposites, polypyrrole, graphene, EMI shielding, Ku-band 


\section{INTRODUCTION}

Electromagnetic interference (EMI) has become a major issue of concern for modern society due to the increased number of electrical and electronic industries. Electromagnetic interference not only damages the electronic instruments but also harms human health. Continuous exposure to electromagnetic radiation can cause many diseases such as leukaemia, miscarriages, and breast cancer. To save society from such harmful electromagnetic radiation, effective shielding from unwanted electromagnetic waves is required. ${ }^{1}$ To date, various studies have been carried out to investigate the dielectric properties and EMI shielding for protecting the environment and sensitive circuits from the electromagnetic radiation generated by telecommunication systems. EMI shielding refers to reflection and/or absorption of electromagnetic radiation by the material that acts as a shielding material that does not allow the penetration of high frequency radiation such as radio waves. The shielding material must be able to absorb the incident radiation in synchronised frequencies, and it is critically important for the EMI materials to be lightweight, flexible, and amenable to fast and low-cost industrial processing.

Metals are the most common materials used for EMI shielding. However, they suffer from disadvantages such as high density, susceptibility to corrosion, complexity and expensive processing. Furthermore, metals mainly reflect radiation and cannot be used in applications where absorption is desired such as in stealth technology. This has led to a great deal of interest in the development of materials that can absorb EMI radiation. In particular, conducting polymers have obtained a special status because of their high EMI shielding efficiency (SE), efficient surface modification and easy formation of composites. ${ }^{2}$ EMI shielding and microwave absorption properties of these polynners can be explained in terms of electrical conductivity and the presence of bound/localized charges (polarons/bipolarons) leading to strong polarisation and relaxation effects. $^{3}$

Polypyrrole (PPy) is a conducting polymer that has attracted a great deal of interest because of its good electrical conductivity, environmental stability, porous nature and easy synthesis. PPy has been used in various applications such as batteries, supercapacitors, sensors, and microwave shielding. Its properties can be further tuned by the control of polymerisation conditions and by using dopants and fillers. In addition, PPy can be used as conducting filler in insulating polymer matrices for the preparation of electrically conducting composites. These composites offer potential use in EMI shields, electronic packaging, display devices and electrodes. ${ }^{4}$ Considerable efforts have been devoted to the fabrication of PPy-based nanocomposites by combining nanoscale carbon fillers with high 
aspect ratios such as carbon nanofibres (CNFs), carbon nanotubes (CNTs), and graphene for EMI shielding absorption. These carbon materials have attracted increasing interest because of their potential applications in ideal absorbers. ${ }^{5,6}$ Among these, graphene has emerged as a new member of carbon allotropes with exceptional carrier mobility and ballistic electron transport properties, making it the prime nanofiller employed in the preparation of nanocomposites for many applications. ${ }^{7,8}$ Graphene is a one-atom-thick planar sheet of $\mathrm{sp}^{2}$-bonded carbon atoms arranged in a hexagonal lattice. It is the thinnest and strongest material. It has remarkable physical, chemical, mechanical, electrical, thermal and microwave absorption properties. In view of the unique structural features of graphene such as its high surface area (theoretical specific surface area (SSA) of $2630 \mathrm{~m}^{2} \mathrm{~g}^{-1}$ ), flexibility, high mechanical strength, chemical stability, and superior electric and thermal conductivities, graphene has been considered to be an ideal material for microwave absorption properties. Graphene nanosheets can be viewed as the building unit, and their reassembly provides opportunities to design and prepare specific structures and hybrids with improved properties for different applications. The most important property of graphene is its electron transport capacity. This means that an electron moves through graphene without much scattering or resistance. It has high electron mobility at room temperature. While its electrical conductivity is much higher than that of copper $(\mathrm{Cu})$, its density is almost four times lower, which is favourable for EMI shielding. ${ }^{9-11}$ Low-cost and solution-processable graphene can be produced from graphene oxide (GO), which in turn is produced from the aggressive oxidation of graphite. Functionalisation of graphene provides the excellent dispersions in aqueous medium and compatibility with the polymer for the production of nanocomposites. ${ }^{12-15}$

The EMI shielding efficiency (SE) of a composite material depends on several important factors including the intrinsic conductivity and aspect ratio of the fillers. Based on these considerations, the composites of conducting polymers and CNTs have become promising materials for achieving high EMI SE. However, the expected EMI SE has not been achieved so far because the heterogeneous interface between the polymer and CNT components of the nanocomposite negatively affects the EMI SE. ${ }^{2,16}$ To overcome this problem, composites based on graphene nanosheets (GNS) have been studied for EMI shielding. ${ }^{17,18}$ Liang et al. prepared graphene/epoxy composites and studied the composites that show a low percolation threshold of 0.52 volume $\%$. The highest EMI SE of $-21 \mathrm{~dB}$ was measured in the X-band. ${ }^{19}$ Eswaraiah et al. fabricated the functionalised graphene/PVDF composite foam and obtained EMI SE values for the composite at higher volume fractions of f-GNS/PVDF that were in the $21-23 \mathrm{~dB}$ range. ${ }^{20}$ Conducting polymers are suitable polymers for further increasing the conductivity of such graphene-based nanocomposites to improve the EMI 
shielding. However, only a few brief reports are available on their microwave absorption characteristics. $^{21}$

In this paper, we report the potential of PPy-coated GNS as possible microwave absorbers. The nanoporous PPy coating may also act as a functional handle for providing good dispersibility and processability of GNS due to the better interaction with host matrix. PPy/graphene nanocomposites were synthesised by in-situ chemical polymerisation to investigate the electrical conductivity, dielectric properties and electromagnetic interference shielding. A high value of EMI SE at a sufficiently low loading of GNS in PPy shows that composites exhibit an absorption-dominant mechanism and can be used as lightweight, effective EMI shielding or microwave absorption materials for protecting electronic devices and components used in satellite communications from electromagnetic radiation in the $\mathrm{K}_{\mathrm{u}}$ band, which is the frequency range from 12.4 to $18 \mathrm{GHz}$ used for the Broadcast Satellite Service (BSS) downlinks. This band is used for domestic TV programs.

\section{EXPERIMENTAL}

\subsection{Materials}

Pyrrole (purity 99\%, mol.wt. $\left.67.09 \mathrm{~g} \mathrm{~mol}^{-1}\right)$, hydrazine monohydrate $\left(\mathrm{N}_{2} \mathrm{H}_{4}\right.$ mol.wt. $\left.32.042 \mathrm{~g} \mathrm{~mol}^{-1}\right)$, and potassium permanganate $\left(\mathrm{KMnO}_{4}\right.$ mol.wt. $158.03 \mathrm{~g}$ $\left.\mathrm{mol}^{-1}\right)$ were supplied by Sigma-Aldrich. Sulphuric acid $\left(\mathrm{H}_{2} \mathrm{SO}_{4}\right.$ mol.wt. $98.079 \mathrm{~g}$ $\left.\mathrm{mol}^{-1}\right)$, nitric acid $\left(\mathrm{HNO}_{3}\right.$ mol.wt. $\left.69.71 \mathrm{~g} \mathrm{~mol}^{-1}\right)$, sodium nitrate $\left(\mathrm{NaNO}_{3}\right.$, mol.wt. $\left.84.9947 \mathrm{~g} \mathrm{~mol}^{-1}\right)$, hydrogen peroxide $\left(\mathrm{H}_{2} \mathrm{O}_{2}\right.$, mol.wt. $\left.34.0147 \mathrm{~g} \mathrm{~mol}^{-11}\right)$ and natural purified graphite were obtained from Merck Ltd., India. Ferric chloride $\left(\mathrm{FeCl}_{3}\right.$, mol.wt. $162.20 \mathrm{~g} \mathrm{~mol}^{-1}$ ) and ethanol (mol.wt. $46.07 \mathrm{~g} \mathrm{~mol}^{-1}$ ) were procured from Himedia. All chemicals were of AR grade and were used as received except for pyrrole, which was distilled under reduced pressure and kept below $4^{\circ} \mathrm{C}$ prior to use for synthesis. Deionised water was used in all syntheses.

\subsection{Synthesis of PPy/GNS Nanocomposites}

GO was synthesised from natural purified graphite by the Hummers method, ${ }^{22,23}$ and GNS were prepared by exfoliation of GO ${ }^{24-27}$ GNS was functionalised by acid treatment to obtain a surface suitable for making chemical interactions with polymers. ${ }^{28,29}$ The preparation of GO and GNS and the functionalisation of GNS were conducted as in our previously reported work. ${ }^{21} \mathrm{PPy} / \mathrm{GNS}$ nanocomposites were synthesised by an in-situ polymerisation of the pyrrole monomer in the presence of functionalised GNS. Different weight percent ratios of GNS to 
pyrrole $(1 \%, 3 \%$ and $5 \%)$ were used. The solution of $0.2 \mathrm{M}$ anhydrous ferrous chloride $\left(\mathrm{FeCl}_{3}\right)$ in $50 \mathrm{ml}$ of deionised water was divided into two parts. Next, 0.2 $\mathrm{M}$ pyrrole and functionalised GNS were mixed and ultrasonicated for $30 \mathrm{~min}$. After ultrasonication, one part of the $0.2 \mathrm{M}$ anhydrous ferrous chloride $\left(\mathrm{FeCl}_{3}\right)$ was added dropwise to the ultrasonicated mixture and kept stirring for $5 \mathrm{~h}$ at $5^{\circ} \mathrm{C}$ to improve the obtained yield. Then, the solution was kept overnight and diluted with deionised water and filtered until the filtrate became colourlessi. Finally, the filtrate was washed with ethanol and dried in a vacuum oven at $810^{\circ} \mathrm{C} . .^{30-35}$ The other part of the $0.2 \mathrm{M}$ anhydrous ferrous chloride $\left(\mathrm{FeCl}_{3}\right)$ was added dropwise to a $0.2 \mathrm{M}$ pyrrole monomer solution without the use of GNS to prepare pure PPy in a similar way. The schematic illustrations of the preparation of PPy/GNS nanocomposites and their EMI shielding measurements are shown in Scheme 1.

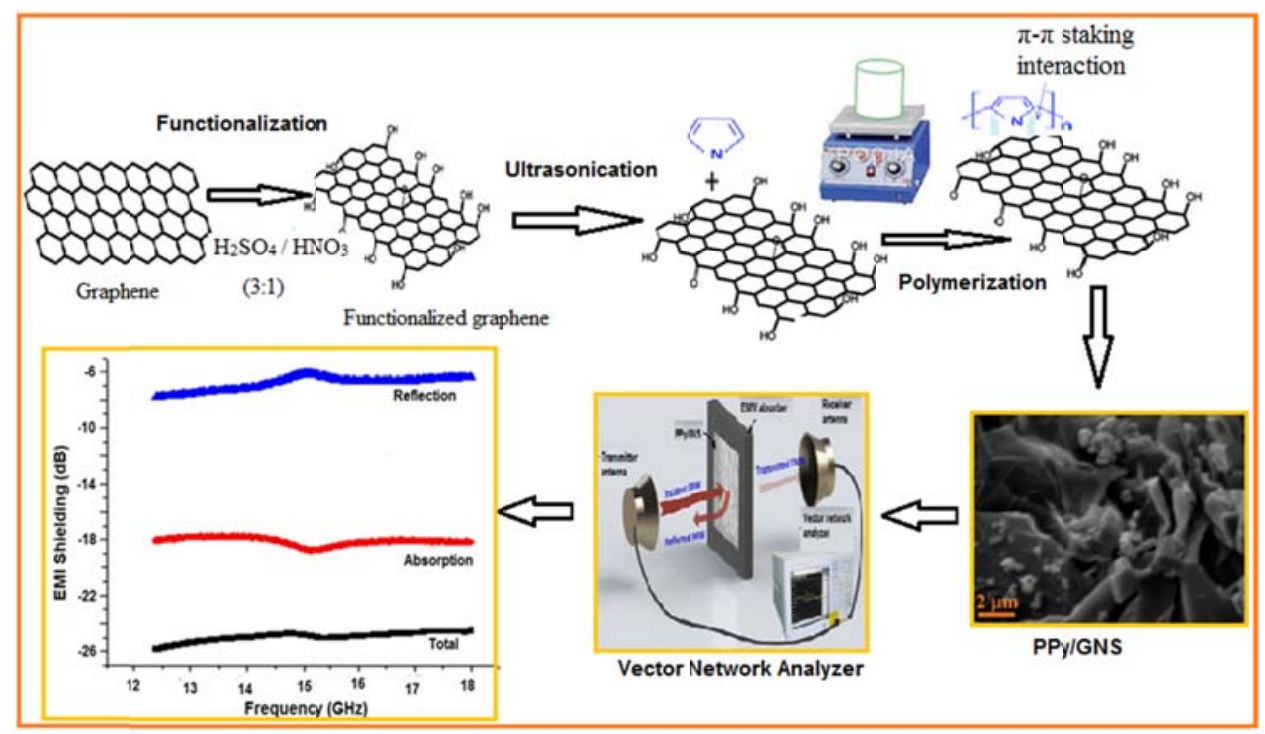

Scheme 1: Schematic of preparation of PPy/GNS nanocomposites and EMI shielding measurement.

\subsection{Instrumental analysis}

SEM images were recorded using a Carl Zeiss EVO-18 scanning electron microscope. A UV 240 Shimadzu automatic double beam spectrograph was used to record the UV-Visible spectra. Fourier transform infrared (FTIR) spectra were recorded using the $\mathrm{KBr}$ pellet technique in the $400-4000 \mathrm{~cm}^{-1}$ wavelength range with the Thermo Nicolet, Avatar 370 infrared spectrophotometer. The electrical conductivity of the compressed pellets of PPy/GNS nanocomposites was determined using the four-probe resistivity technique. The pellets were prepared using a hydraulic press (Kimaya Engineers, India) by applying a pressure of 5000 
$\mathrm{kg} \mathrm{cm}^{-2}$. Electromagnetic interference shielding, permittivity and dielectric loss measurements were carried out with a Vector Network Analyzer in the microwave range of $12.4-18 \mathrm{GHz}\left(\mathrm{K}_{\mathrm{u}}\right.$ band) using Agilent Technologies (E8362B).

\section{RESULTS AND DISCUSSION}

\subsection{Scanning Electron Microscopy (SEM)}

To study the surface morphology, SEM images of functionalised GNS, pure PPy and PPy/GNS nanocomposites as shown in Figure 1(a-e) were obtained using scanning electron microscopy. Functionalised graphene exhibits a shiny black appearance with a metallic lustre. The transparency of these functionalised GNS confirms the successful exfoliation of graphene sheets and indicates that GNS is composed of a few individual graphene sheets. ${ }^{36}$ As shown in Figure 1(a), GNS typically have a curved layer-like structure that is irregular in form and with the lateral size of several micrometres with many leaf-like veins on the surface that arise from the crumpling and scrolling of graphene sheets with porous structure. Graphene sheets are rippled and wrinkled, with dimensions of several hundred $\mathrm{nm}$ to several $\mu \mathrm{m}$. Such cloth-like graphene layers were often observed in previous studies. ${ }^{37,38}$ PPy shows granular-like morphology (Figure 1(b)). The observed individual granules were nearly spherical and showed a close packing and high porosity. It appears that such sphericulates grow on one another, forming a continuous structure. ${ }^{39}$ The bulk sphericulate structure typically appears similar to a "cauliflower." The macro-granular structure formed by the aggregation of small globular structures is probably due to an increased interchain interaction. ${ }^{40,41}$ Figures $1(\mathrm{c}-\mathrm{e})$ show a uniform layer coating of the $\mathrm{PPy} / \mathrm{GNS}$ nanocomposites and the sphere-like morphology of PPy particles on the GNS sheets. The uniform spherical morphology builds up PPy with some pores throughout the GNS. PPy as a monomer diffused into the graphene nanosheets during in situ polymerisation and polymerised on the surface of graphene nanosheets layer by layer. In such nanocomposites, graphene sheets act as the electron acceptor while PPy serves as the electron donor. 

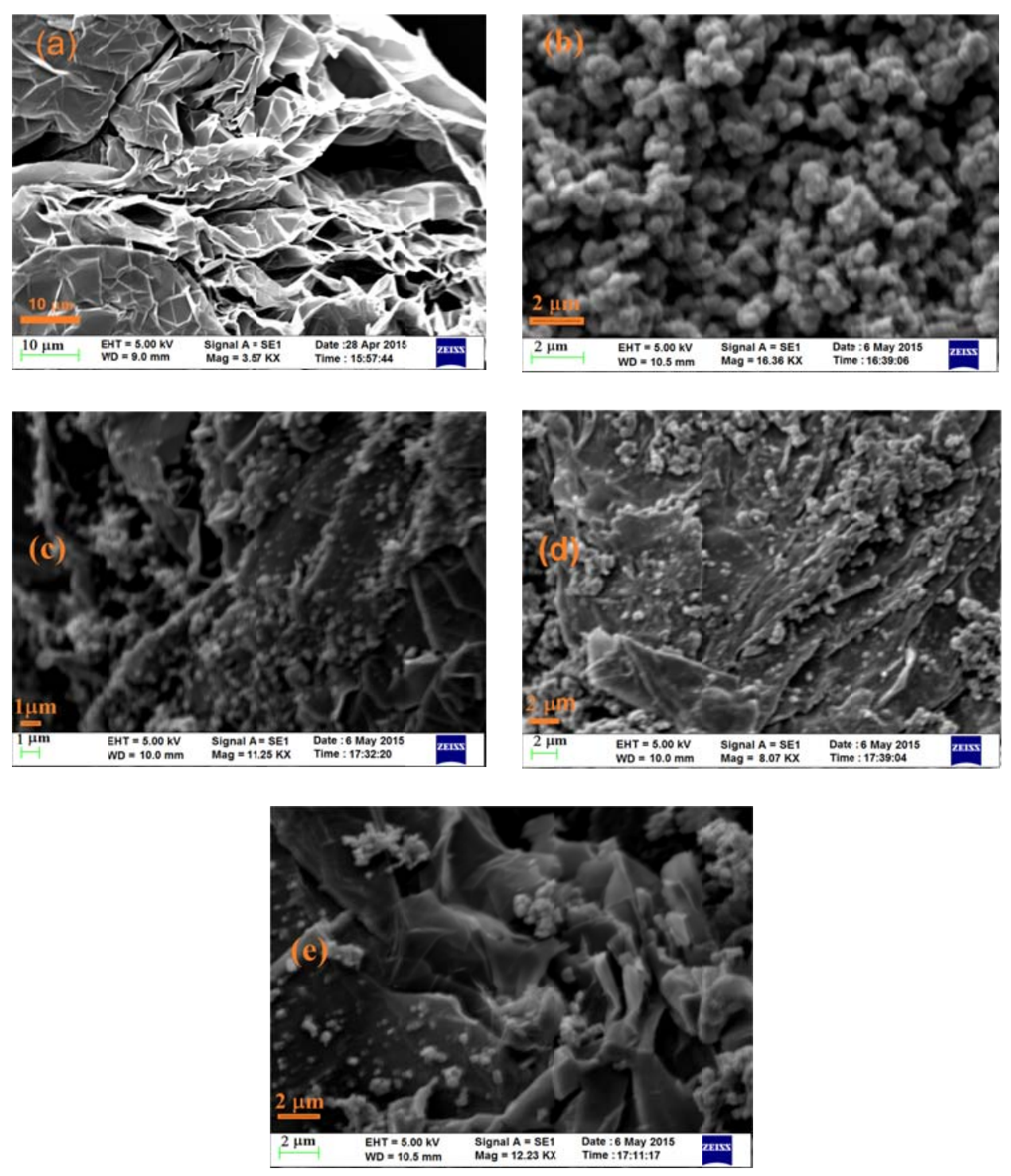

Figure 1: SEM images of (a) functionalised graphene, (b) pure PPy, (c) PPy/1\%GNS, (d) PPy $/ 3 \%$ GNS, and (e) PPy $/ 5 \%$ GNS.

\subsection{UV-Visible Spectroscopy}

Figure 2 shows the UV-Visible absorption spectra for pure PPy and different $\mathrm{PPy} / \mathrm{GNS}$ compositions. PPy showed three bands, one at $305 \mathrm{~nm}$ corresponding to the $\pi \rightarrow \pi^{*}$ inter band transition and the other bands at 440 and $755 \mathrm{~nm}$, which were assigned to the polaron- $\pi^{*}$ and bipolaron band transitions of PPy. 
For PPy/GNS nanocomposites, the polaron- $\pi^{*}$ band shift owing to the extended PPy chains indicates good conjugation; hence, the high degree of doping and conductivity for the PPy/GNS nanocomposites confirms the interaction between the GNS sheets and PPy spheres. In the UV-visible spectra of PPy/GNS, the GNS dispersion shows a peak at $282 \mathrm{~nm}$ and a tiny shoulder at $350 \mathrm{~nm}$ that are attributed to the $\pi-\pi^{*}$ transitions of aromatic C-C bonds and the $n-\pi^{*}$ transitions of $\mathrm{C}=\mathrm{O}$ bonds, respectively. ${ }^{42}$ The absorption peak for GNS shifts toward the higher wavelength with the addition of PPy. This means that the energy of the $\pi-\pi^{*}$ transitions of aromatic $\mathrm{C}=\mathrm{C}$ bonds in GNS is changed considerably by the addition of PPy; the changes are expected to arise from the $\pi$ - $\pi$ stacking between PPy and GNS.

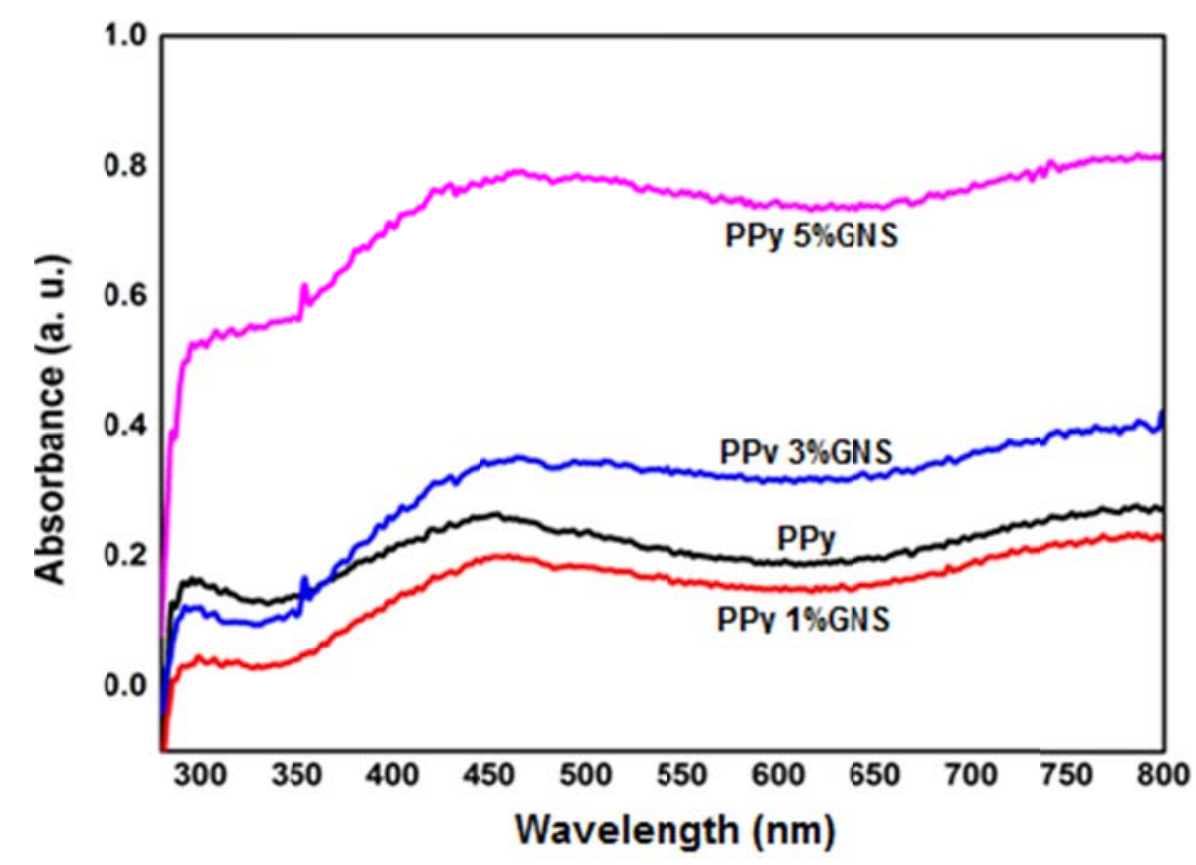

Figure 2: UV-VIS spectra of PPy/GNS nanoomposites.

\subsection{FTIR Spectroscopy}

Figure 3 shows the FT-IR spectra for PPy and PPy/GNS nanocomposites. For pure PPy, the characteristic peaks at 1549.70 and $1300.75 \mathrm{~cm}^{-1}$ correspond to the stretching vibrations of $\mathrm{C}=\mathrm{C}$ and $\mathrm{C}-\mathrm{N}$ of $\mathrm{PPy}$, respectively. The peak at $1180 \mathrm{~cm}^{-1}$ is attributed to the breathing vibration of the pyrrole ring. The band of $\mathrm{C}-\mathrm{H}$ and N-H in-plane deformation vibration is located at $1042.86 \mathrm{~cm}^{-1}$, while the band of C-H out-of-plane deformation vibration was found at $915.23 \mathrm{~cm}^{-1}$. The expected peak of the $\mathrm{S}=\mathrm{O}$ attributed to the stretching vibration of the sulphonic 
group at $1183 \mathrm{~cm}^{-1}$ could not be clearly observed due to overlap with the pyrrole ring vibration at $1180.51 \mathrm{~cm}^{-1} .{ }^{43}$ For PPy/GNS nanocomposites, a broad peak at approximately $3436.08 \mathrm{~cm}^{-1}$ and peaks at $1629.97,1297.56$, and $1038.88 \mathrm{~cm}^{-1}$ are attributed to the $\mathrm{O}-\mathrm{H}$ stretching vibration and the carbonyl $(\mathrm{C}=\mathrm{O}), \mathrm{C}-\mathrm{O}-\mathrm{C}$ and $\mathrm{C}-\mathrm{O}$ stretching vibrations, respectively. It is possible to recognise the characteristic peaks of PPy at 1544.22, $1297.56 \mathrm{~cm}^{-1}$ in the FTIR spectra of the nanocomposites, indicating that polymerisation has occurred in the presence of graphene. Some of the nanocomposite peaks are shifted to lower energies owing to the interactions between graphene and PPy. The graphene peaks are slightly specific in the nanocomposites because of the low concentration of graphene in PPy. ${ }^{9}$

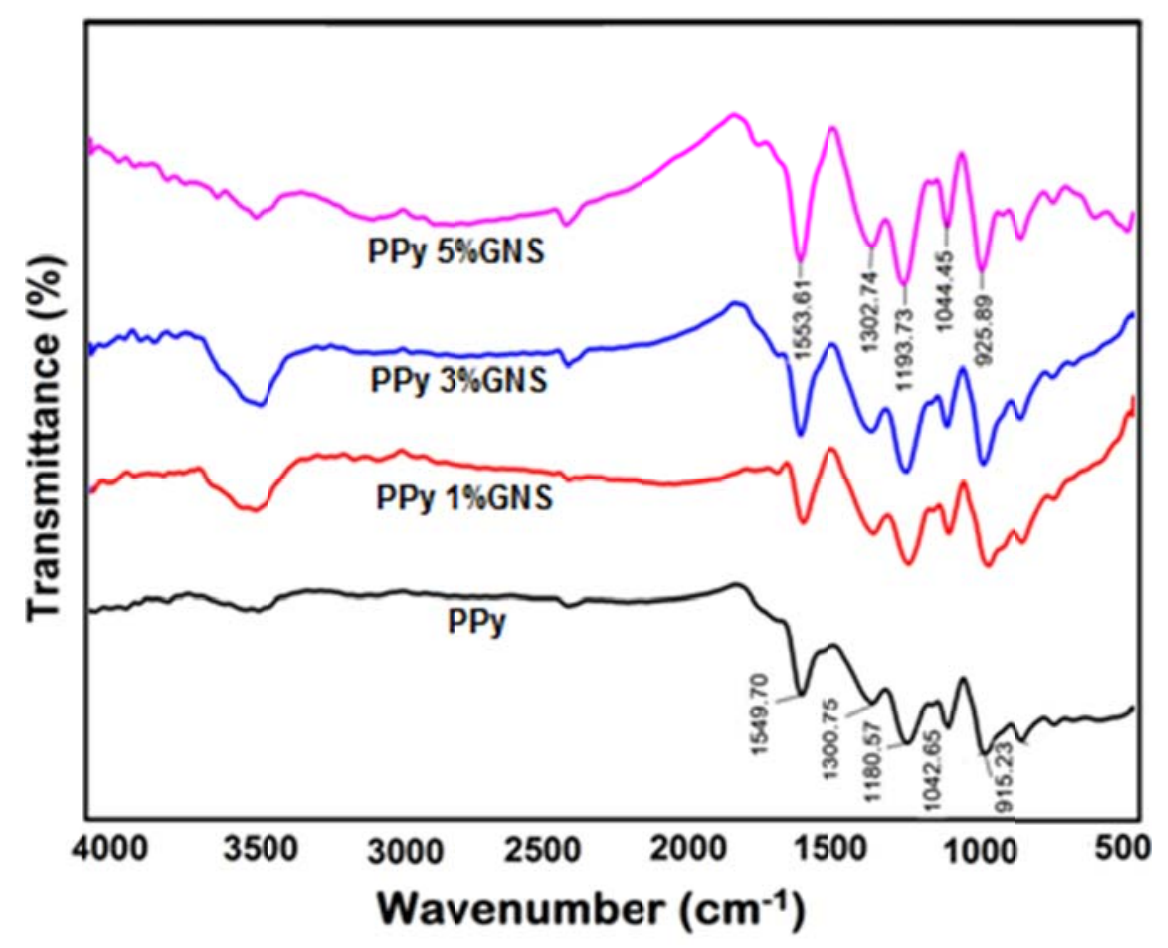

Figure 3: FTIR spectra of PPy/GNS nanocomposites.

\subsection{Electrical Conductivity}

The electrical measurements of the PPy/GNS nanocomposites were carried out using the 4-probe method. For electrical measurements, the PPy/GNS nanocomposites in the powder form were made into a pellet and were placed under a 4-probe resistivity set up for determination of electrical conductivity. It has been observed that the electrical conductivity of the nanocomposites 
increased almost linearly with increasing temperature, as shown in Figure 4, indicating semiconducting behaviour similar to that of pure PPy. The deposition of PPy over the GNS facilitates the electron transfer process between the PPy channels. The remarkable enhancement of electrical conductivity potentially endows the lightweight PPy/GNS with good EMI shielding characteristics. Examination of Figure 4 shows that the highly temperature-dependent nature of the nanocomposites at low graphene content can be related to the dominance of the tunnelling mechanism when the number of graphene sheets is insufficient to allow physical contact between the sheets. Comparison of the PPy composites with $1 \%, 3 \%$ and $5 \%$ GNS shows that the highest conductivity of $2.34 \mathrm{~S} \mathrm{~cm}^{-1}$ was observed for $1 \%$ GNS content. The extent of the temperature dependence became less noticeable when the GNS content increased above the percolation threshold. PPy/GNS nanocomposites showed a non-linear increase in electrical conductivity as a function of GNS concentration. Above $3 \mathrm{wt} \%$ GNS in PPy, the electrical conductivity was found to be almost constant. Specifically, $3 \mathrm{wt} \%$ GNS in conducting polymer (PPy) matrix is the percolation threshold for electrical conductivity of conducting PPy/GNS nanocomposites. At this point, the physical contacts among the fillers are well developed and the "contact" conductivity dominates over the tunnelling mechanism. The slight decrease in conductivity with increasing temperature is evidence of the prominent role of the contact conductivity in these composites.

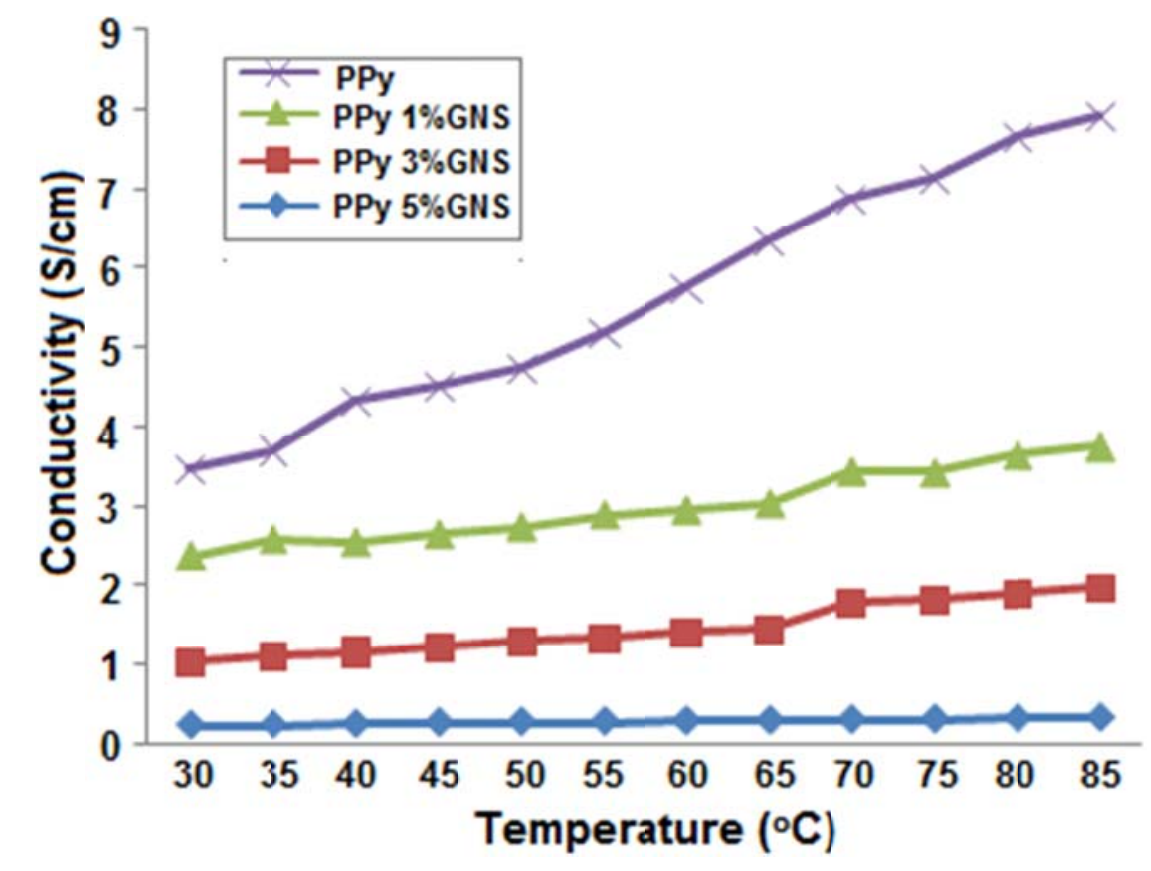

Figure 4: Temperature dependence of D.C. conductivity of PPy/GNS nanocomposites. 


\subsection{Complex Permittivity and Permeability}

Permittivity and dielectric loss measurements were carried out using a vector network analyser in the microwave range of $12.4-18 \mathrm{GHz}\left(\mathrm{K}_{\mathrm{u}}\right.$ band). To investigate the possible microwave absorption mechanism, we determined the real and imaginary parts of the complex permittivity $\left(\varepsilon^{\prime}, \varepsilon^{\prime \prime}\right)$ and permeability $\left(\mu^{\prime}, \mu^{\prime \prime}\right)$ from the scattering parameters using the Nicolson-Ross-Weir (NRW) method. The incident and transmitted travelling waves inside a vector network analyser can be represented by complex scattering parameters or the $\mathrm{S}$ parameters, i.e., $S_{11}$ or $S_{22}$ and $S_{12}$ or $S_{21}$, which are related to the electromagnetic characteristics of permittivity and permeability. The NRW technique is formulated from the set of equations related to these $\mathrm{S}$ parameters and is useful for providing direct calculations for both permittivity and permeability. Our results show that permittivity values exhibit a trend of decreasing with the increase in frequency. This can be attributed to the decreasing ability of the dipoles present in the system to maintain the in-phase movement with the rapidly oscillating electric vector of the incident EM wave. At low frequencies, the electric dipoles have sufficient time for aligning with the field before the field changes its direction; consequently, the dielectric constant is high. However, at higher frequencies, the dipoles fail to follow the rapidly changing electric vector; consequently, the dielectric constant value decreases. The results also revealed that the dielectric constant $\left(\varepsilon^{\prime}\right)$ as well as dielectric loss $\left(\varepsilon^{\prime \prime}\right)$ values of the composite exhibit a noticeable enhancement upon addition of GNS, as shown in Figures 5(a) and 5(b).

As the GNS concentration increases, the permittivity of the composites increases as well. This is due to the increase in the space charge build up caused by the interfacial polarisation. The presence of doping-induced localised charges (polarons or bipolarons) on the PPy backbone gives rise to strong polarization effects. Furthermore, in conducting polymers, the space charge formation due to the conductivity difference between the ordered or highly conducting (crystalline or metallic) islands and the electrically insulating amorphous matrix contributes toward the interfacial polarisation. The complete polarisation effects and associated loss mechanisms are responsible for the high dielectric constant values. Similarly, the associated relaxation effects lead to enhancement of the $\varepsilon^{\prime \prime}$ values. The real and imaginary permittivity in PPy/GNS at the lower concentration is independent of frequency. For higher concentrations, the permittivity decreases with increasing frequency. The real permeability values of composites with higher GNS concentrations were found to be higher. This was due to the improvement of the magnetic properties along with the reduction of eddy current losses. Similarly, the composites with higher GNS concentration show higher magnetic losses. The introduced magnetic properties also lead to a 
better matching of the input impedance along with the reduction of the skin depth and enhanced absorption of the electromagnetic microwaves. Figures 6(a) and 6 (b) show the real part of permeability $\left(\mu^{\prime}\right)$ for a PPy/GNS composite of approximately 1.2 ; this value may have been obtained owing to the ferric sulphate used in the Ppy synthesis. The results shows that the imaginary part $\left(\mu^{\prime \prime}\right)$ is in the negative region for the $1 \%$ GNS concentration of the PPy/GNS composite, which means that the radiation passes through the material without any absorption. It is clear that dielectric loss is the most important contribution, rather than the magnetic loss. Despite the very small value, their permeability plot showed that the composites also have magnetic property characteristics.
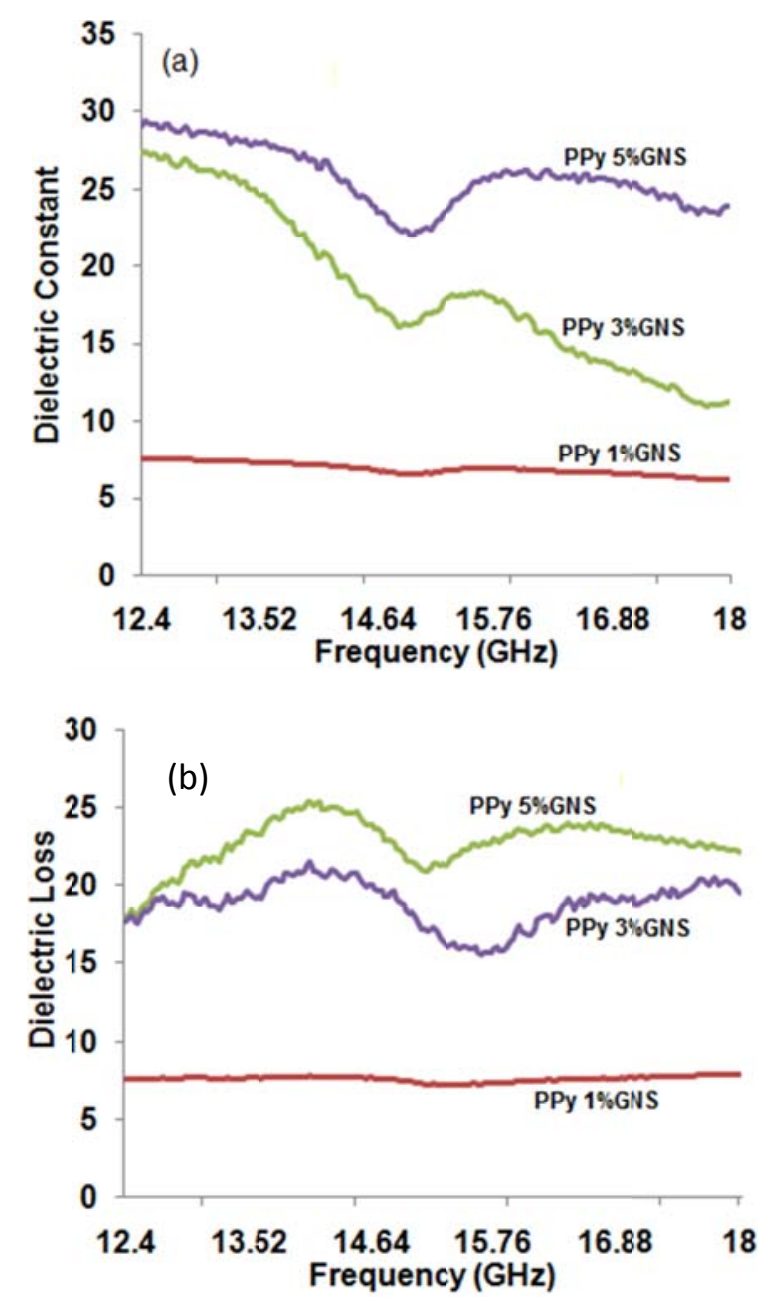

Figure 5: Frequency dependence of (a) dielectric constant, (b) dielectric loss of $\mathrm{PPy} / \mathrm{GNS}$ nanocomposites. 

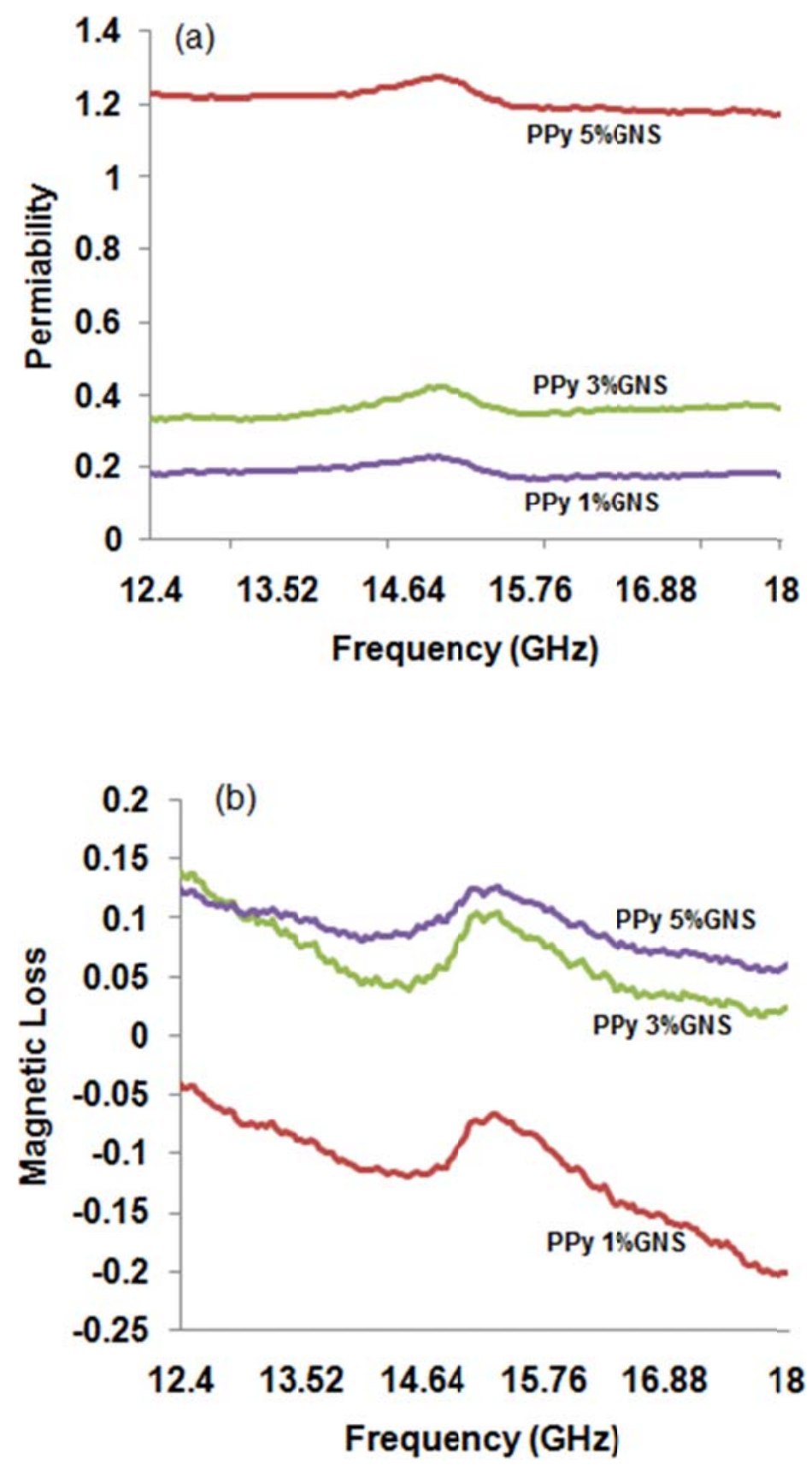

Figure 6: Frequency dependence of (a) permeability and (b) magnetic loss of PPy/GNS nanocomposites. 


\subsection{Electromagnetic Shielding Effectiveness}

The electromagnetic interference shielding effectiveness is defined as the logarithmic ratio of the incoming (Pi) power input to the outgoing power (Po) of radiation. The efficiency of any shielding material is expressed in decibels (dB). The higher the decibel level of electromagnetic interference shielding effectiveness, the lesser will be the energy transmitted through the shielding material. ${ }^{20}$ The shielding effectiveness (SE) of a shielding material is equal to the sum of the absorption factor, the reflection factor and the multiple reflections. ${ }^{44}$

The synthesised composite material consists of PPy/GNS, and the polymer matrix containing GNS enhances the interfacial polarisation and the effective anisotropy energy of the sheets; this contributes to more scattering. The materials also show a high shielding effectiveness compared to conventional materials. Addition of GNS as filler in PPy shows better microwave absorption properties that strongly depend on the volume fraction of the filler. Therefore, the high value of EMI SE is dominated by absorption rather than reflection. The EMI shielding effectiveness in the composites increases with increasing GNS content. With the increase in GNS from $1 \%$ to $5 \%$, the volume resistivity of the composites decreases and the shielding and effectiveness increase. The number of percolating networks increases with the increase in the GNS amount. The conductive networks formed due to the dispersion of GNS behave as conductive meshes. With the increase in GNS loading, the size of the conductive mesh decreases, acting as a barrier to incident electromagnetic radiation and giving rise to a higher EMI SE. This is because the electrical conductivity of a composite tends to increase with increasing GNS content, and upon the action of electromagnetic radiation, an induction current generated on the interface or in the interior of the sample produces a reversal electromagnetic field, leading to the increase in surface reflection attenuation of electromagnetic waves and consequently increasing the EMI shielding effectiveness of the composite. ${ }^{45}$

The attenuation of the incident wave increases by increasing the absorption cross section and scattering cross section of the absorbent particle. The attenuation of the incident wave energy increases as well. A larger specific surface area of the GNS effectively increases the plane wave absorption cross section and scattering cross section of absorbing particles so that the electromagnetic wave loss is increased. It is well known that the total shielding effectiveness of PPy is dominated by absorption phenomena due to the presence of localised charges (polarons and bipolarons) leading to the strong divergence and relaxation effects. The PPy coating on the GNS can dominate the polarisation, and the functional groups of functionalised GNS give rise to the electromagnetic radiation absorption. The functional groups of GNS are also responsible for the absorption 
due to the increase in the content of GNS functional groups that are responsible for the absorption of electromagnetic radiation. The effect of conductivity on reflection and absorption loss (EMI SE) of a material depends on many factors such as conductivity, dielectric constant, aspect ratio, state of dispersion of conductive fillers, and thickness of shielding materials. Among all of these factors, conductivity is the primary factor for an EMI shielding material. In the case of a conductive material such as metal, EMI SE is mainly due to reflection of EM radiation, but for a material such as a conductive composite, EMI SE is mainly due to radiation absorption. The reflection shielding effectiveness $\left(\mathrm{SE}_{\mathrm{R}}\right)$, absorption shielding effectiveness $\left(\mathrm{SE}_{\mathrm{A}}\right)$ and total shielding effectiveness $\left(\mathrm{SE}_{\mathrm{T}}\right)$ of PPy/GNS nanocomposites as a function of frequency are shown in Figures $7(\mathrm{a}-\mathrm{c})$, for $1 \%, 3 \%$ and $5 \%$ GNS loading in $\mathrm{PPy}$, respectively. The $\mathrm{SE}_{\mathrm{R}}$ is nearly linear for each composition in the entire frequency range of measurement and shows a negligible change even with the increase in GNS loading. The $\mathrm{SE}_{\mathrm{A}}$ is increased from 8 to $18 \mathrm{~dB}$ with the increase in GNS loading from 1 to $5 \mathrm{wt} \%$.

The experimental results show that absorption is the primary shielding mechanism and that reflection is the secondary shielding mechanism. The $\mathrm{SE}_{\mathrm{T}}$ of the nanocomposites as a function of frequency shows that the nature of $\mathrm{SE}_{\mathrm{T}}$ for each composition is nearly linear with frequency, but the $\mathrm{SE}_{\mathrm{T}}$ of the composite is found to increase with increased GNS loading. The total shielding effectiveness values for PPy/GNS were 11, 24 and 26 for 1, 3, and $5 \mathrm{wt} \%$ GNS loading, respectively. For PPy/GNS composites, a high value of EMI SE is obtained that is higher than the value of the EMI shielding effectiveness $(20 \mathrm{~dB})$ required for commercial applications. Such a high value of EMI SE at a sufficiently low loading of GNS shows the efficiency of the compounding technique. The EMI $\mathrm{SE}$ results show that composites have an absorption-dominant mechanism and can be used as lightweight effective EMI shielding or $\mathrm{Ku}$ band microwave absorption materials for protection of electronic devices and components from electromagnetic radiation. 

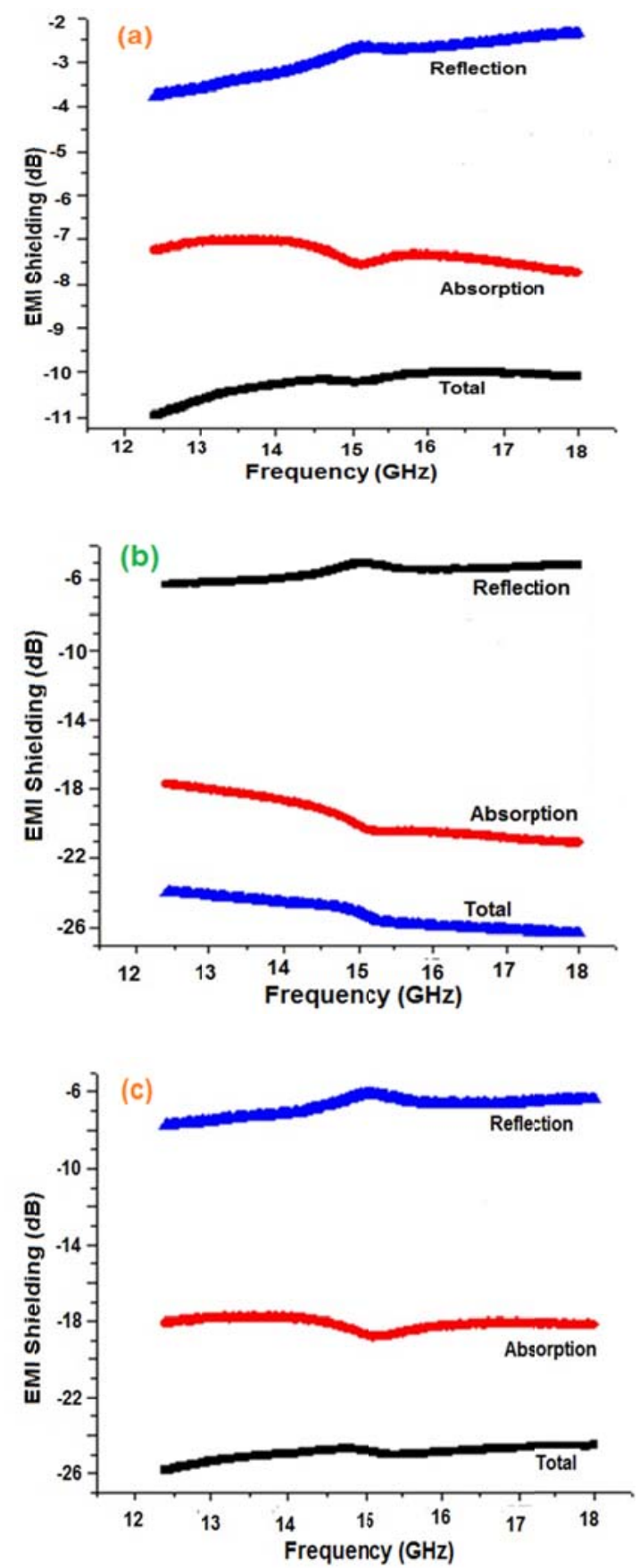

Figure 7: EMI shielding effectiveness for (a) PPy/1\%GNS, (b) PPy/3\%GNS, and (c) PPy $/ 5 \%$ GNS nanocomposites. 


\section{CONCLUSION}

Highly conducting polypyrrole (PPy)/graphene (GNS) nanocomposites were prepared by in situ polymerisation with different concentrations of functionalised GNS (1\%, 3\% and 5\%). UV-VIS and FTIR spectra of composites confirmed the significant interaction between PPy and GNS. SEM images show a thick and uniform coating of PPy over the surface of the individual GNiS. PPy/GNS nanocomposites showed a semiconducting nature similar to that of PPy but with improved properties for EMI shielding. The electromagnetic shielding effectiveness (SE) and dielectric constant of the composites were found to increase with increasing GNS content, and the high obtained value of EMI SE makes this composite useful as a lightweight EMI shielding material for satellite commercial applications.

\section{ACKNOWLEDGEMENT}

Author Subhash B. Kondawar acknowledges University Grants Commission (UGC), India for providing financial support to carry out the research work through a Major Research Project Grant No. F. 39- 540/2010 (SR).

\section{REFERENCES}

1. Gupta, T. K. et al. (2013). Improved nanoindentation and microwave shielding properties of modified MWCNT reinforced polyurethane composites. R. Soc. Chem. Adv., 1(32), 9138-9163, http://dx.doi.org/ 10.1039/C3TA11611E.

2. Im, J. S. et al. (2010). Enhanced adhesion and dispersion of carbon nanotube in PANI/PEO electrospun fibers for shielding effectiveness of electromagnetic interference. Coll. Surf. A, 364, 151-157, http://dx.doi.org/10.1016/j.colsurfa.2010.05.015.

3. Saini, P. et al. (2009). Polyaniline-MWCNT nanocomposites for microwave absorption and EMI shielding. Mater. Chem. Phys., 113, 919926, http://dx.doi.org/10.1016/j.matchemphys.2008.08.065.

4. Reung-U-Rai, A. et al. (2008). Synthesis of highly conductive polypyrrole nanoparticles via microemulsion polymerization. J. Metal. Mater. Miner, 18(2), 27-31.

5. Cao, M. S. et al. (2010). The effects of temperature and frequency on the dielectric properties, electromagnetic interference shielding and microwave-absorption of short carbon fiber/silica composites. Carbon, 4(8), 788-796. 
6. Zhou, H. et al. (2013). Synthesis and electromagnetic interference shielding effectiveness of ordered mesoporous carbon filled poly(methyl ethacrylate) composite films. R. Soc. Chem. Adv., 3, 23715-23721, http://dx.doi.org/10.1039/C3RA44267E.

7. Yousefi, N. et al. (2014). Highly aligned graphene/polymer nanocomposites with excellent dielectric properties for high-performance electromagnetic interference shielding. Adv. Mater., 26, 5480-5487, 10.1002/adma.201305293.

8. Udmale, V. et al. (2013). Development trends in conductive nanocomposites for radiation shielding. Orien. J. Chem., 29(3), 927-936.

9. Oliveira, D. et al. (2013). Supercapacitors from free-standing polypyrrole/graphene nanocomposites. J. Phys. Chem., 117(20), 1027010276, http://dx.doi.org/10.1021/jp400344u.

10. Hong, S. K. et al. (2012). Electromagnetic interference shielding effectiveness of monolayer graphene. Nanotechnol., 23(45), 455704455708, http://dx.doi.org/10.1088/0957-4484/23/45/455704.

11. Chen, T. et al. (2014). Enhanced electromagnetic wave absorption properties of polyanilline-coated $\mathrm{Fe}_{3} \mathrm{O}_{4} /$ reduced grapheme oxide nanocomposites. J. Mater. Sci.: Mater. Electro., 25, 3664-3673, http://dx.doi.org/10.1007/s10854-014-2073-1.

12. Bai, H. et al. (2009). Non-covalent functionalization of graphene sheets by sulfonated polyaniline. Chem. Commun., 13, 1667-1669, http://dx.doi.org/10.1039/B821805F.

13. Hanifah, M. F. R. et al. (2015). Effect of reduction time on the structural, electrical and thermal properties of synthesized reduced graphene oxide nanosheets. Bull. Mater. Sci., 38(6), 1569-1576, http://dx.doi.org/ 10.1007/s12034-015-0970-4.

14. Kumar, D. et al. (2015). A 1 V supercapacitor device with nanostructured graphene oxide/polyaniline composite materials. Bull. Mater. Sci., 38(6), 1507-1517, http://dx.doi.org/10.1007/s12034-015-0966-0.

15. Hao, Q. et al. (2011). Morphology-controlled fabrication of sulphonated graphene/polyaniline nanocomposites by liquid/liquid interfacial polymerization and investigation of their electrochemical properties. Nanotech. Res., 4, 323-333, http://dx.doi.org/10.1007/s12274-0100087-4.

16. Kumaran, R. et al. (2016). Enhanced electromagnetic interference shielding in Au-MWCNT composite nanostructure dispersed PVDF thin films. J. Phy. Chem. C, 20, 13771-13778, http://dx.doi.org/10.1021/ acs.jpcc.6b01333.

17. Shen, B. et al. (2016). Compressible graphene coated polymer foams with ultralow density for adjustable electromagnetic interference (EMI) shielding. ASC App. Mat. Int., 8, 8050-8057, http://dx.doi.org/ 10.1021/acsami.5b11715 
18. Pawar, S. et al. (2016). Tailored electrical conductivity, electromagnetic shielding and thermal transport in polymeric blends with graphene sheets decorated with nickel nanoparticles. Phys. Chem. Chem. Phys, 17, 14922-14930, http://dx.doi.org/10.1039/C5CP00899A.

19. Liang, J. et al. (2009). Electromagnetic interference shielding of graphene/epoxy composites. Carbon, 47, 922-925, http://dx.doi.org/ 10.1016/j.carbon.2008.12.038.

20. Eswaraiah, V., Sankaranarayanan, V. \& Ramaprabhu, S. (2011). Functionalized graphene-PVDF foam composites for EMI shielding. Macromol. Mater. Eng., 296, 894-899.

21. Modak, P., Kondawar, S. B. \& Nandanwar, D. V. (2015). Synthesis and characterization of conducting polyaniline/graphene nanocomposites for electromagnetic interference shielding. Proc. Mater. Sci., 10, 588-594.

22. Hummers, W. S. \& Offeman, R. E. (1958). Preparation of graphitic oxide. J. Am. Chem. Soc., 80, 1339-1341, http://dx.doi.org/10.1021/ ja01539a017.

23. Xie, F., Qi, S. H. \& Wu, D. (2016). A facile strategy for the reduction of graphene oxide and its effect on thermal conductivity of epoxy based composites. Exp. Polym. Lett., 10(6), 470-478, http://dx.doi.org/10.3144/ expresspolymlett.2016.45.

24. Singh, A. P. et al. (2012). Phenolic resin-based composite sheets filled with mixtures of reduced graphene oxide, $\Upsilon-\mathrm{Fe} 2 \mathrm{O} 3$ and carbon fibers for excellent electromagnetic interference shielding in the X-band. Carbon, 50, 3868-3875, http://dx.doi.org/10.1016/j.carbon.2012.04.030.

25. Li, M. et al. (2015). Excellent electrochemical performance of homogenous polypyrrole/graphene composites as electrode material for supercapacitors. J. Mater. Sci. Mater. Electr., 26(1), 485-492, http://dx.doi.org/10.1007/s10854-014-2425-x.

26. Lim, Y. S. et al. (2013). Preparation and characterization of polypyrrole/graphene nanocomposite films and their electrochemical performance. J. Polym. Res., 20, 156-161, http://dx.doi.org/10.1007/ s10965-013-0156-y.

27. Bose, S. et al. (2011). Electrochemical performance of a graphenepolypyrrole nanocomposite as a supercapacitor electrode. Nanotechnol., 22, 295202-295211, http://dx.doi.org/10.1088/0957-4484/22/29/295202.

28. Cordero, N. A. \& Alonso, J. A. (2007). The interaction of sulphuric acid with graphene and formation of adsorbed crystals. Nanotechnol., 18(48), 485705-4857113, http://dx.doi.org/10.1088/0957-4484/18/48/485705.

29. Bautista-Flores, C., Sato-Berrú, R. Y. \& Mendoza, D. (2015). Doping graphene by chemical treatments using acid and basic substances. $J$. Mater. Sci. Chem. Eng., 3, 17-21, http://dx.doi.org/10.4236/msce. 2015.310003. 
30. Basnayaka, P. A. et al. (2013). Graphene/polypyrrole nanocomposites as electrochemical supercapacitor electrode: Electrochemical impedence studies. Graphene, 2(2), 81-87, http://dx.doi.org/10.4236/graphene. 2013.22012.

31. Yang, Z. et al. (2014). Polypyrrole/graphene oxide composites electrode for high energy density supercapacitors. Adv. Mater. Res., 904, 146-149, http://dx.doi.org/10.4028/www.scientific.net/AMR.904.146.

32. Pham, H. D. et al. (2012). Synthesis of polypyrrole- reduced grapheme oxide composites by in situ photopolymerization and its application as a supercapacitor electrode. J. Chem. Eng., 29(1), 125-129, http://dx.doi.org/10.1007/s11814-011-0145-y.

33. Yao, W. \& Lu, Y. (2014). Preperation of graphene/Fe3o4/polypyrrole nanocomposite and its adsorption for $\mathrm{Cr}(\mathrm{VI})$ ions. Adv. Mater. Res., 905, 61-64, http://dx.doi.org/10.4028/www.scientific.net/AMR.905.61.

34. Konwer, S. Boruah, R. \& Dolui, S. K. (2011). Studies on conducting polypyrrole/graphene oxide composites as supercapacitor electrode. $J$. Electro. Mater., 40(11), 2248-2252, http://dx.doi.org/10.1007/s11664011-1749-z.

35. Whitby, R. L. D. et al. (2011). Morphological effects of single-layer grapheme oxide in the formation of covalently bonded polypyrrole composites using intermediate diisocyanate chemistry. J. Nanopart. Res., 13, 4829-4837, http://dx.doi.org/10.1007/s11051-011-0459-z.

36. Dubin, S. et al. (2010). A one-step, solvothermal reduction method for producing reduced graphene oxide dispersions in organic solvents. Am. Chem. Soc. Nano., 4(7), 3845-3852, http://dx.doi.org/10.1021/ nn100511a.

37. Patole, A. S. et al. (2012). Self assembled graphene/carbon nanotube/polystyrene hybrid nanocomposite by in situ micromulsion polymerization. Europ. Polym. J., 48, 252-259, http://dx.doi.org/ 10.1016/j.eurpolymj.2011.11.005.

38. Harish, C. et al. (2012). Synthesis of polyaniline/graphene nanocomposites and its optical, electrical and electrochemical properties. Adv. Sci. Eng. Med., 5, 140-149, http://dx.doi.org/10.1166/asem. 2013.1237.

39. Chitte, H. K. et al. (2011). Synthesis of polypyrrole using ammonium peroxy disulfate (APS) as oxidant together with some dopants for use in gas sensors. Mater. Sci. Appl., 2, 1491-1498, http://dx.doi.org/10.4236/ msa.2011.210201.

40. Abdulla, H. S. \& Abbo, A. I. (2012). Optical and electrical properties of thin films of polyaniline and polypyrrole. Int. J. Electrochemi. Sci., 7, 10666-10678.

41. Eisazadeh, H. (2007). Studying the characteristics of polypyrrole and its composites. World J. Chem., 2(2), 67-74. 
42. Trang, L. K. H. et al. (2012). Preparation and characterization of graphene composites with conducting polymers. Polym. Int., 61, 93-98, http://dx.doi.org/10.1002/pi.3152.

43. Yalcinkaya, S. et al. (2010). Electrochemical synthesis and characterization of polypyrrole/chitosan composite on platinum electrode: Its electrochemical and thermal behaviours. Carbohydr. Polym., 79, 908-913, http://dx.doi.org/10.1016/j.carbpol.2009.10.022.

44. Al-Saleh, M. H. \& Sundararaj, U. (2009). Electromagnetic interference shielding mechanisms of CNT/polymer composites. Carbon, 47, 17381746, http://dx.doi.org/10.1016/j.carbon.2009.02.030.

45. Muhammad, F. \& Syed, K. (2014). Ku-band EMI Shielding effectiveness and dielectric properties of polyaniline- $\mathrm{Y}_{2} \mathrm{O}_{3}$ composites. Polym. Sci. Ser. A, 56(3), 366-372, http://dx.doi.org/10.1134/S0965545X14030055. 